\title{
Norrie disease
}

INSERM

\section{Source}

INSERM. (1999). Orphanet: an online rare disease and orphan drug data base. Norrie disease. ORPHA:649

Norrie disease (ND) is a rare X-linked genetic vitreoretinal condition characterized by abnormal retinal development with congenital blindness. Common associated manifestations include sensorineural hearing loss and developmental delay, intellectual disability and/or behavioral disorders. 